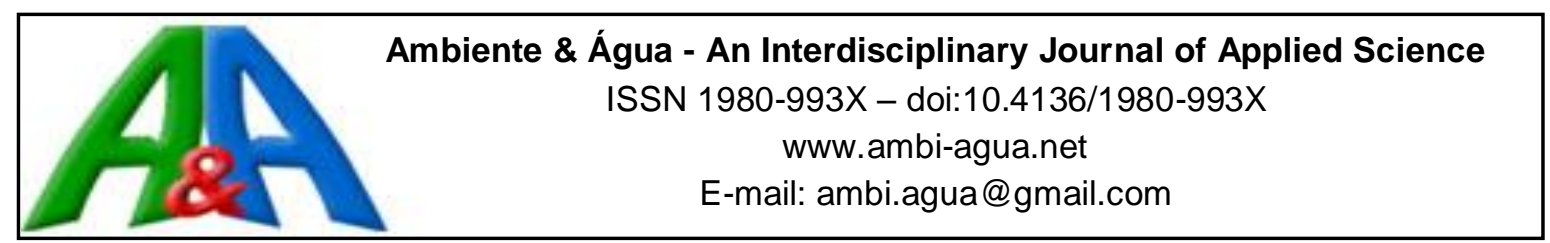

\title{
Aves em quintais e a relação com os proprietários de residências em área urbana da cidade de Jacareí, São Paulo, Brasil
}

\author{
doi:10.4136/ambi-agua.1916
}

Received: 26 Aug. 2016; Accepted: 10 Oct. 2016

\author{
Solange Fátima Gonçalves ${ }^{1 *}$; Maria Cecília Barbosa de Toledo ${ }^{1}$ \\ ${ }^{1}$ Universidade de Taubaté (UNITAU), Taubaté, SP, Brasil \\ Programa de Pós-Graduação em Ciências Ambientais \\ *Autor correspondente: e-mail: solangefatimag@gmail.com, \\ mceciliabt@gmail.com
}

\section{RESUMO}

O objetivo deste estudo foi avaliar a diversidade de aves em quintais e associar com a percepção dos proprietários sobre as aves que ocorrem em seus quintais e investigar se as características do habitat do quintal, decorrentes de modificações realizadas pelo proprietário, influenciam o número de espécies, a abundância e a diversidade de aves. Para isso, foram avaliados 31 quintais de casas localizadas na área central da cidade de Jacareí, SP. O levantamento das espécies de aves do quintal foi realizado usando o método de pontos de contagem. As características do quintal foram amostradas sendo: tamanho, número de árvores e número de plantas nativas e exóticas, bem como, foram registradas as modificações que o proprietário fez no quintal, tais como porcentagem impermeabilizada do solo, presença de animais de estimação e alimentadores para aves. Um questionário foi usado para saber informações sobre o proprietário, como espécies de aves observadas, idade, tempo de residência e sobre os inconvenientes causados pelas as aves. O levantamento resultou em 31 espécies $\left(\mathrm{H}^{\prime}=2,51\right)$ e a pesquisa realizada através do conhecimento dos proprietários resultou em 58 espécies $\left(\mathrm{H}^{\prime}=3,56\right)$. O pombo doméstico e o pardal foram apontados como as espécies mais inconvenientes. As modificações nos quintais feitas pelos proprietários podem influenciar positivamente e negativamente a comunidade de aves nos quintais, sendo que (1) a presença de alimentadores resultou num maior número médio de espécies de aves, (2) os quintais com menor área impermeabilizada obteveram maior abundância de aves, e (3) a presença de gatos livres nos quintais foi desfavorável à diversidade de aves. Os resultados indicam que é possível melhorar o habitat do quintal com pequenas ações locais que tem como objetivo atrair as aves e colaborar com a sua conservação no ambiente urbano.

Palavras-chave: aves urbanas, diversidade, quintais residenciais.

\section{Backyard bird community and the relationship with residence owners in the urban area of Jacarei city, Vale do Paraíba, São Paulo, Brazil}

\section{ABSTRACT}

The aim of this study was to evaluate the diversity of birds in backyards and to associate their presence with the perception of the owners about the birds that occur in their backyards. The study also investigated whether the characteristics of the backyard habitat, due to modifications made by the owner influence the total number of species, abundance and 
diversity of birds. The backyards of 31 houses in the urban area of Jacareí city, SP were evaluated. The method of count point was used to survey the species of birds. Backyard characteristics such as size, number of trees, and number of native and exotic plants were noted, and modifications to the backyard by the owner, such as the percentage of impermeabilized soil, the presence of pets, and bird feeders were noted. A questionaire was used to find out information regarding the owner such as the bird species observed, age, length of residence, and inconveniences caused by the birds. The survey found 31 bird species $\left(\mathrm{H}^{\prime}=2.51\right)$ and the knowledge of the owners resulted in 58 species $\left(\mathrm{H}^{\prime}=3.56\right)$. The species pointed out as the most inconvenient were the domestic pigeon and the sparrow. Modifications in backyards made by the owners can influence the bird community both positive and negatively, in that (1) the presence of feeders increases the average number of bird species, (2) the backyard smaller impermeabilized area resulted in a greater abundance of birds, and (3) free cats in the backyards were unfavorable to the diversity of birds. The results showed that, in order to attract the birds and assist in their conservation in the urban environment, is possible to improve backyard habitats with small local actions.

Keywords: backyards, diversity, urban birds.

\section{INTRODUÇÃO}

As cidades abrigam hoje mais da metade da população mundial (Butler e Spencer, 2010). Em geral, as áreas arborizadas nas grandes cidades estão intimamente ligadas ao nível social, pois quanto maior o poder aquisitivo mais arborizado é o local, tais como casas com grandes jardins, grandes quintais, ou residências que eram antigas chácaras (Luchiari, 2001; Fuller et al., 2007; Toledo et al., 2011; Belaire et al., 2015).

Por outro lado, projetos atuais destinados a conjuntos habitacionais possuem área verde limitada, com pequenos quintais, e em muitos casos apresentam apenas corredores. Somado a isso, muitos proprietários procuram aproveitar ao máximo o terreno com construções (Ferreira, 2011) o que resulta num afastamento das pessoas da natureza. Isso tem levado o ser humano a perder a afinidade com o meio ambiente e não se reconhecer como sendo parte da natureza (Miller, 2005).

A Classe Aves é um dos grupos mais utilizados em estudos na avaliação de mudanças no ecossistema por ter elevado número de espécies presentes em praticamente todos os ambientes e constituindo-se em importantes bioindicadores de qualidade ambiental (Bagliano, 2012). Em pesquisa realizada no Condado de Cook, na cidade de Chicago, Illinois, Estados Unidos da América, os moradores dos bairros avaliados admitiram valorizar as aves porque reconheciam que além da beleza elas eram parte daquele ecossistema (Belaire et al., 2015).

A observação de aves pode ser uma ferramenta para trazer as pessoas de volta ao contato com a natureza, estimulando a consciência de conservação da biodiversidade (Müller et al., 2010; Oppliger et al., 2016). Os quintais podem ser um instrumento de sensibilização e incentivo à percepção das pessoas em relação à importância das aves quanto aos serviços ecossistêmicos (Galluzzi et al., 2010). Algumas modificações e ações realizadas nos quintais das residências pelos proprietários podem contribuir com o incremento de espécies de aves (Beumer e Martens, 2014) como a suplementação alimentar, que pode tornar o quintal mais atraente (Parsons et al., 2006). Assim como o plantio de arvores fornece abrigo, locais para nidificação e descanso contribuindo para visitação das aves nos jardins (Fernández Juricic, 2000; Melles et al., 2003; Beumer e Martens, 2014).

Este estudo teve por objetivo avaliar a percepção dos proprietários de residências urbanas quanto à diversidade de aves existentes em seus quintais e relacionar as características dos 
quintais resultantes das modificações realizadas pelo proprietário com a riqueza, abundância e diversidade de aves.

\section{MATERIAL E MÉTODOS}

\section{1. Área de estudo e seleção dos quintais}

O município de Jacareí (23¹7’55.62” S; 4557’58.51”O) localiza-se no início da Bacia do Rio Paraíba do Sul, na região Metropolitana do Vale do Paraíba, São Paulo, Brasil. Apresenta área territorial de $464.272 \mathrm{~km}^{2}$, com população de 211.214 habitantes e densidade demográfica de aproximadamente 454,94 hab./ $\mathrm{km}^{2}$ (IBGE, 2010). Apresenta $79 \%$ de área rural, sendo o restante dividido em 14\% de área urbanizada e $7 \%$ área de várzea antropizada, utilizada para agricultura. O município está inserido no domínio de Mata Atlântica (IBGE, 2012), o clima é mesotérmico, com verão quente e inverno seco, sendo os meses mais frios e secos de julho a agosto e os meses mais quentes e chuvosos de novembro a março (Álvares et al., 2013 ).

Foram selecionados 17 bairros dentro da área urbana (Figura 1), incluindo a área central, de Jacareí. Nessa área a maior parte das edificações residenciais e comerciais ainda são predominantemente horizontais. Foram estudadas 31 residências, selecionadas em função da permissão dos proprietários (Comitê de Ética da Unitau; processo n.: 113961/2014).

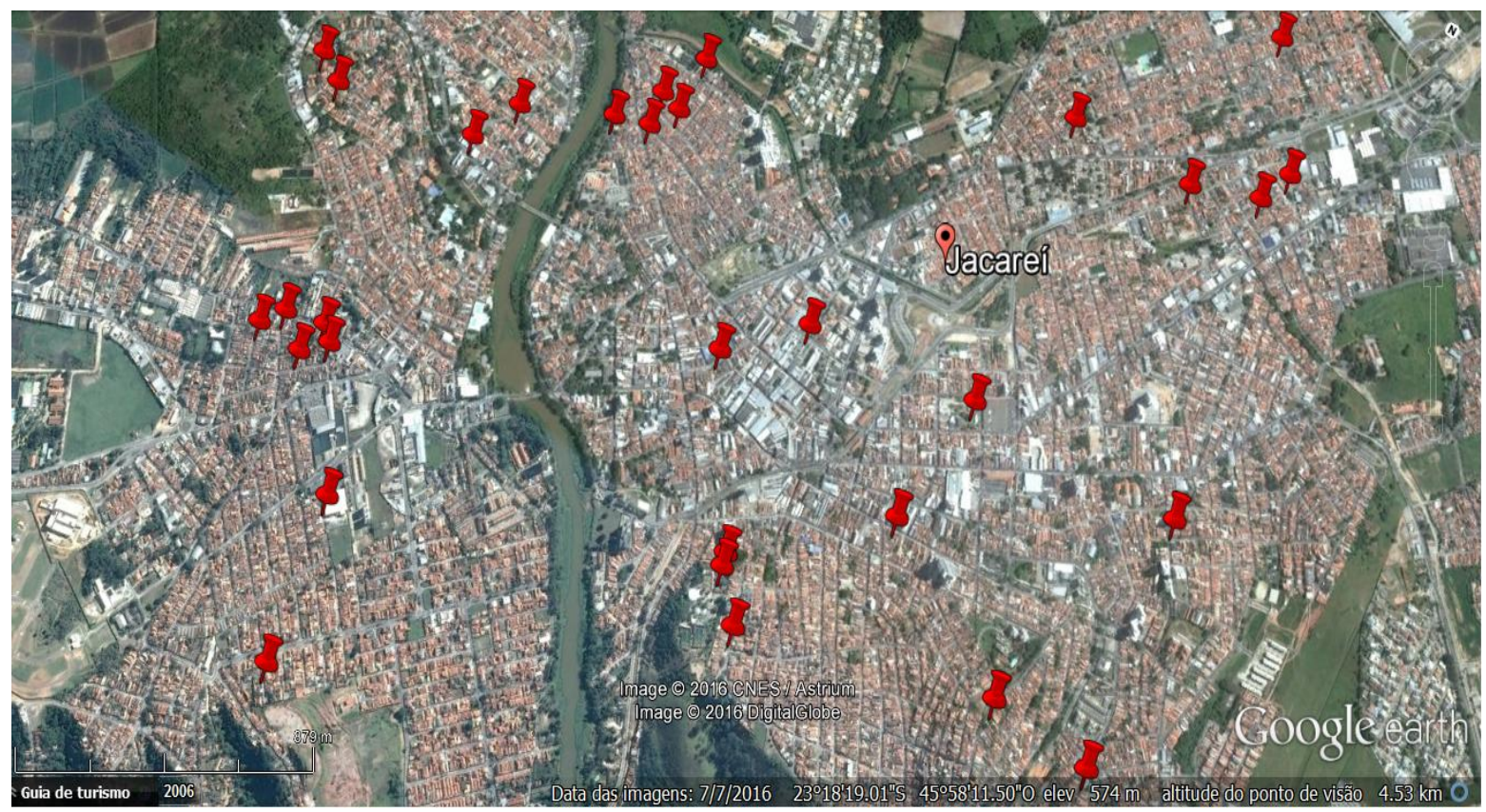

Figura 1. Imagem da área urbana do município de Jacareí. Os pontos em vermelho indicam os quintais estudados.

Fonte: Googleearth.com.br

\subsection{Caracterização dos quintais a partir das decisões do proprietário}

Inicialmente, com utilização de trena foi tomada a medida da área do quintal. Quanto à vegetação foi realizada a identificação das espécies vegetais usando o manual de identificação de espécies de Lorenzi et al. (2013) e ainda por meio de perguntas ao proprietário.

Assim, para cada residência, foi elaborada uma lista contendo as espécies vegetais e a classificação entre nativas e exóticas, bem como uma estimativa de porcentagem de área impermeabilizada nas categorias: $0 \%$; $\langle 25 \% ; 25 \%$ a $50 \%$ e $>50 \%$ de impermeabilização. 
Foram registradas e avaliadas a presença de animais domésticos nos quintais (cães, gatos, aves de gaiola e galinhas), sendo também avaliada a disponibilidade de alimentos complementares para atração das aves, como comedouros ou restos de alimentos jogados no quintal, a presença de água e número e espécies de plantas que compunham a vegetação do quintal.

\subsection{Levantamento da avifauna}

O censo da avifauna foi realizado com base no trabalho de Daniels e Kirkpatrick (2006) e portanto foi utilizado o método de pontos de contagem. Foi escolhido um ponto que permitisse a clara visibilidade das aves e maximizasse a visibilidade de todo quintal, onde o pesquisador permaneceu por 20minutos. Os mesmos pontos foram usados em todas as visitas. O levantamento foi visual e auditivo, visto que em todas foi realizada a gravação simultânea para posterior análise e confirmação da identificação das vocalizações.

A coleta de informações foi realizada no período de Janeiro à Julho de 2015. O horário estabelecido foi pela manhã entre $6 \mathrm{~h} 30$ e $10 \mathrm{~h} 30$ ou no final da tarde entre $16 \mathrm{~h} 30$ e $18 \mathrm{~h} 00$. Os quintais foram escolhidos de acordo com a permissão do proprietário. Todos os contatos foram registrados em planilhas de campo, onde foram registradas: nome da espécie presente, número de indivíduos e item alimentar, quando possível. Somente foram registrados os indivíduos que ocupavam efetivamente o quintal, portanto, as espécies predominantemente aéreas, tal como andorinha, foram excluídas. Também foram registrados a data, o horário de início e fim da observação e as condições ambientais, como presença de vento, nebulosidade, chuva fraca, calor ou frio e ruído de fundo. As observações não foram realizadas quando vento e nebulosidade impediram a precisa identificação visual e auditiva. Por uma questão probabilística cada residência foi amostrada três vezes. Foi mantido um intervalo aproximado de 30 dias entre as visitas realizadas em uma mesma residência. Ao final foi elaborada uma lista das espécies de aves registradas,

A identificação das espécies e quantificação de indivíduos foi realizada com o auxílio de binóculos 8X42, gravador digital, máquina fotográfica e guias ornitológicos. A nomenclatura das espécies citadas segue a lista das aves do Brasil atualizada em 2015 pelo Comitê Brasileiro de Registros Ornitológicos (PIACENTINI et al., 2015).

\subsection{Levantamento da avifauna a partir da percepção do proprietário}

Foram realizadas entrevistas com base em um questionário contendo perguntas específicas abertas e fechadas. Inicialmente eram perguntas que caracterizavam o morador, como tempo de residência no local e idade do proprietário. Ainda no mesmo questionário foram realizadas perguntas quanto ao conhecimento das aves por parte do proprietário e sobre vegetação. Para esta avaliação contamos com o auxílio de guias ornitológicos, contendo fotos de aves frequentemente vistas na região, seguindo a metodologia de Beumer e Martens, (2014) em que o proprietário poderia manipular o livro e calmamente identificar as espécies que o mesmo já havia observado no quintal. Quando os participantes indicavam que os mesmos não estavam confiantes em sua identificação as aves não eram incluídas na lista. Foi também questionado se (1) o proprietário atribuía às aves algum tipo de problema existente em seu quintal, (2) a opinião quanto a fatores positivos e negativos em relação à presença das aves e (3) a manutenção da vegetação também foram registradas.

A grande maioria dos proprietários soube identificar as aves por meio do nome popular, com isso quando ficavam em dúvida em relação à foto do guia ornitológico escreviam o nome pelo qual conheciam determinada ave. Portanto, em alguns casos, foi possível o registro somente em nível de família ou morfo-espécie, como no caso das corujas e pica-paus. 


\subsection{Análise dos dados}

Com base no censo da vegetação foi calculada a diversidade de plantas usando o índice de Shannon. Para avaliar se o tamanho da área influenciava no número de espécies de plantas e arvores foi usado a correlação linear de Pearson (r).

A partir do levantamento de aves foram calculados: abundâncias absoluta e relativa de cada espécie, número de espécies, Índice de Shannon e equabilidade para descrever a comunidade de aves (Magurran, 2013).

A partir da lista de espécies de aves obtida a partir dos proprietários foi aplicado o índice de diversidade de Shannon.

Também se realizou uma análise quanto a porcentagem de área impermeabilizada por meio da comparação usando ANOVA one way entre a riqueza de espécies nos quintais com $0 \%$; $<25 \%$; $25 \%$ a $50 \%$ e $>50 \%$ de área impermeabilizada seguida do teste de Dunn. A riqueza de aves foi comparada entre os proprietários que mantinham e não mantinham alimentadores. Para tanto foi usado teste Mann-Whitney (U).

Realizou-se a comparação entre a abundância, riqueza e diversidade de aves nos quintais que tinham animais de estimação, como cães, gatos, aves de gaiola e galinhas. Em função das diferentes categorias de animais de estimação registradas nos quintais foram considerados quatro grupos sendo: presença de gato, gato+cachorro, cachorro e cachorro+passarinho, sendo utilizado o teste Mann-Whitney (U) para comparação.

\section{RESULTADOS E DISCUSSÃO}

A maior porcentagem dos entrevistados eram moradores antigos no local e residiam na propriedade em média há 27 anos. Do total de participantes, dois $(6,4 \%)$ não mencionaram suas idades, quanto aos demais, um (3,2\%) tinha idade abaixo de 30 anos; quatro $(12,9 \%)$ apresentaram faixa etária entre 30 e 40 anos; cinco $(16,1 \%)$ apresentaram idade entre 40 a 50 anos; cinco (16,1\%) idade entre 50 e 60 anos; nove (29\%) entre 60 a 70 anos de idade, e cinco $(16,1 \%)$ idade acima de 70 anos. Assim de modo geral mais de $65 \%$ dos proprietários tinham idade acima de 50 anos.

Do total de proprietários questionados sobre se as árvores eram problema no quintal, 22 (71\%) disseram que as árvores presentes em seus quintais não eram um problema, pois atraiam as aves, proporcionando abrigo e alimentação. Dentre os que consideraram um problema ter árvores em seus quintais, quatro (12,9\%), citaram como justificativas: sujeira e atração de pombo-doméstico ou pardal, que são vistos como pragas. Deixaram a questão sem responder cinco proprietários $(16,1 \%)$.

A área média dos 31 quintais estudados foi de 255,4 $\pm 362,6 \mathrm{~m}^{2}$ (valor máx: $2.000 \mathrm{~m}^{2} \mathrm{e}$ valor mín: $32 \mathrm{~m}^{2}$ ). Em relação às plantas, foram registradas 115 espécies nos quintais, sendo $69 \%$ exóticas, $29 \%$ nativas e três espécies de origem incerta (Quadro 1). As plantas mais comuns foram: laranja, $14.8 \%$ e limão 8,7\% (Citrus spp.); jabuticaba (Myrcia cauliflora Berg) 12\%; acerola (Malpighia emarginata DC) 10\%; mangueira (Mangífera indica L.) 8,7\%; mamão (Carica papaya L) 8,7\%; grama (Paspalum notatum Flügge.) 8,7\%; goiabeira (Psidium guajava L.) 7,8\%; heliconia (Heliconia sp.) 6,9\%; hibisco (Malvaviscus arboreus Cav.) 6,9\%; gêneros variados de palmeiras (Arecaceae) 22,6\%, além de outras espécies registradas em menor número. Assim, observou-se uma proporção de 2:1 de plantas exóticas nos quintais avaliados. O número médio de espécies vegetais por quintal foi de $10,6 \pm 3,8$ resultando em uma diversidade de Shannon=3.3. O tamanho da área dos quintais explicou $40 \%$ do número de espécies de plantas presentes $(\mathrm{r}=0,40 ; \mathrm{p}=0,02)$ e $47 \%$ do número de árvores $>4 \mathrm{~m}(\mathrm{r}=0,47 ; \mathrm{p}=0,00)$. 
Quadro 1. Lista de espécies de plantas registradas em 31 quintais de residências na área urbana de Jacareí, SP.

\begin{tabular}{|c|c|c|c|}
\hline Família & Espécie & Nome Vulgar & Origem \\
\hline Anacardiaceae & Anacardium occidentale L. & Caju & $\mathrm{n}$ \\
\hline Asparagaceae & Dracaena fragrans( $L$.$) Ker Gawl.$ & Pau d'água & $\mathrm{e}$ \\
\hline Asparagaceae & Agave sp. & Agave & $\mathrm{e}$ \\
\hline Asparagaceae & Cordyline fruticosa (L.) A. Chev. & Dracena vermelha & $\mathrm{e}$ \\
\hline Asparagaceae & Chlorophytum Comosum & Clorofito & $\mathrm{e}$ \\
\hline Cecropiaceae & Cecropia pachystachya Trécul & Embaúba & $\mathrm{n}$ \\
\hline Cucurbitáceas & Sechium edule (Jacq.) & Chuchu & $\mathrm{e}$ \\
\hline Curcubitáceas & Momordica charantia & Goia & $\mathrm{e}$ \\
\hline Fabaceae & Arachis repens & Grama amendoim & $\mathrm{n}$ \\
\hline Iridaceae & Gladiolus hortulanus L. H. Bailey & Palma & $\mathrm{e}$ \\
\hline Leguminosae & Caesalpinia pulcherrima $S w$. & Flamboyant mirim & e \\
\hline Lythraceae & Lagerstroemia indica $\mathrm{L}$. & Resedá & e \\
\hline Malvacea & Hibiscus acetosella Welw. & Vinagreira & $\mathrm{e}$ \\
\hline Malvacea & Malva L. & Malva & $\mathrm{e}$ \\
\hline Meliaceae & Cedrela sp. & Cedro & $\mathrm{n}$ \\
\hline Myrtaceae & Syzygium aromaticum & Cravo & e \\
\hline Phytolaccaceae & Petiveria tetrandra Gomez & Guiné & $\mathrm{i}$ \\
\hline Poaceae & Brachiaria plantaginea & Capim marmelo & e \\
\hline Rubiaceae & Ixora macrothyrsa Teijsm. E Binn. & Ixora-rei & $\mathrm{e}$ \\
\hline Rutaceae & Citrus spp. & Laranjeira & $\mathrm{e}$ \\
\hline Acanthaceae & Odontonema tubaeforme (Bertol.) Kuntze & Odontonema & e \\
\hline Acanthaceae & Pachystachys lutea Nees & Camarãozinho & $\mathrm{e}$ \\
\hline Acanthaceae & Justicia carnea Lindl. & Jacobinia & $\mathrm{n}$ \\
\hline Acanthaceae & Ruellia brevifolia (Pohl) C. Ezcurra & Pingo-de-sangue & $\mathrm{n}$ \\
\hline Agavaceae & Yucca guatemalensis & Yuca & $\mathrm{e}$ \\
\hline Amaranthaceae & Amaranthus viridis $\mathrm{L}$. & Caruru & $\mathrm{e}$ \\
\hline Anacardiaceae & Mangífera indica $\mathrm{L}$. & Mangueira & $\mathrm{e}$ \\
\hline Annonaceae & Annona squamosa L. & Fruta do conde & e \\
\hline Apiaceae & Foeniculum vulgare Mill. & Funcho & $\mathrm{e}$ \\
\hline Araceae & Anthurium andraeanum Lindl. & Antúrio & e \\
\hline Araceae & Spathiphyllum wallisii Regel & Lírio & $\mathrm{e}$ \\
\hline Araceae & Epipremnum pinnatum $\mathrm{L}$. & Jibóia & $\mathrm{e}$ \\
\hline Araceae & Xanthosoma sagittifolium (L.) & Taioba & $\mathrm{e}$ \\
\hline Araceae & Epipremnum pinnatum & Gibóia & $\mathrm{e}$ \\
\hline Araliaceae & Schefflera actinophylla & Cheflera & e \\
\hline Araucariaceae & Araucaria heterophylla (Salisb.) & Pinheiro & $\mathrm{e}$ \\
\hline Arecaceae & Archontophoenix alexandrae H. Wendl. \& Drude & Palmeira beatriz & e \\
\hline Arecaceae & Dypsis lutescens. H. Wendel & Palmeira areca & $\mathrm{e}$ \\
\hline Arecaceae & Caryota mitis Lour. & $\begin{array}{l}\text { Palmeira rabo de } \\
\text { peixe }\end{array}$ & $\mathrm{e}$ \\
\hline Arecaceae & Roystonea oleracea & Palmeira imperial & e \\
\hline Arecaceae & Latania chinensis & Palmeira-leque & e \\
\hline Arecaceae & Cocos nucifera L. & Palmeira & $\mathrm{e}$ \\
\hline Arecaceae. & Livistona chinensis $R$. & Palmeira & e \\
\hline Asteraceae & Mikania glomerata Spreng. & Guaco & $\mathrm{n}$ \\
\hline Asteraceae & Vernonia condensata Baker & Boldo baiano & $\mathrm{e}$ \\
\hline Asteraceae & Taraxacum officinale Weber & Dente-de-leão & $\mathrm{i}$ \\
\hline Balsaminaceae & impatiens walleriana $L$. & Maria-sem-vergonha & $\mathrm{e}$ \\
\hline Bignoniaceae & Handroanthus avellanedae (Lorentz \& Griseb.) & Ipê roxo & $\mathrm{n}$ \\
\hline Bignoniaceae & Tecoma stans $(\mathrm{L})$. & Ipê-amarelo & $\mathrm{e}$ \\
\hline Bignoniaceae & Tabebuia spp. & Ipê amarelo & $\mathrm{n}$ \\
\hline Bignoniaceae & Tabebuia sp. & Ipê branco & $\mathrm{n}$ \\
\hline Brassicaceae & Brassica oleracea $\mathrm{L}$. & Couve & $\mathrm{e}$ \\
\hline Bromeliaceae & Ananas sativus Schult. & Abacaxizeiro & $\mathrm{n}$ \\
\hline Cactaceae & Rhipsalis teres (Vell.) Steud. & Ripsális & $\mathrm{n}$ \\
\hline
\end{tabular}

Continua.... 


\begin{tabular}{|c|c|c|c|}
\hline \multicolumn{4}{|l|}{ Continuação... } \\
\hline Caricaceae & Carica papaya $\mathrm{L}$ & Mamão & e \\
\hline Cicadaceae & Cycas revoluta & Cica & e \\
\hline Cupressaceae & Thuja sp. & Pinheiro & $\mathrm{e}$ \\
\hline Euphorbiaceae & Phyllanthus tenellus Roxb. & Quebra-pedra & $\mathrm{n}$ \\
\hline Euphorbiaceae & Manihot esculenta Crantz & Mandioca & $\mathrm{n}$ \\
\hline Euphorbiaceae & Codiaeum variegatum (L.) Rumph. & Cróton & e \\
\hline Fabaceae & Hymenaea courbaril $\mathrm{L}$ & Jatoba & $\mathrm{n}$ \\
\hline Fabaceae & Schizolobium parahyba (Vell.) Blake & Guapuruvu & $\mathrm{n}$ \\
\hline Heliconiaceae & Heliconia rostrata Ruiz \&Pav. & Helicônia & $\mathrm{n}$ \\
\hline Hypoxidaceae & Curculigo capitulata (Lour.) Kuntze & Capim-palmeira & $\mathrm{e}$ \\
\hline Iridaceae & Neomarica caerulea (Ker Gawl.) Sprague & Isis & e \\
\hline Lamiaceae & Clerodendrum thomsoniae Balf. & Lágrima -de-cristo & $\mathrm{e}$ \\
\hline Lamiaceae & Plectranthus barbatus Andrews. & Boldo & e \\
\hline Lamiaceae & Mentha sp & Hortelã & $\mathrm{e}$ \\
\hline Lamiaceae & Ocimum basilicum $\mathrm{L}$. & Manjericão & $\mathrm{e}$ \\
\hline Lauraceae & Persea americana Mill. & Abacateiro & $\mathrm{e}$ \\
\hline Lauraceae & Cinnamomum burmanni & Falsa Canela & $\mathrm{e}$ \\
\hline Leguminosae & Cajanus cajan cv. & Feijão gandu & $\mathrm{e}$ \\
\hline Leguminosae & Caesalpinia peltophoroides Benth. & Sibipiruna & $\mathrm{n}$ \\
\hline Lomariopsidaceae & Nephrolepis pectinata (Willd.) Schott & Samambaia paulista & $\mathrm{n}$ \\
\hline Lythraceae & Punica granatum $L$ & Romã & $\mathrm{e}$ \\
\hline Malpighiaceae & Malpighia emarginata DC & Acerola & $\mathrm{e}$ \\
\hline Malvaceae & Malvaviscus arboreus Cav. & Hibisco & $\mathrm{e}$ \\
\hline Malvaceae & Abutilon striatum Dicks. ex Lindl & Lanterna chinesa & $\mathrm{n}$ \\
\hline Melastomataceae & Tibouchina mutabilis (Vell.) & Manaca-da-serra & $\mathrm{n}$ \\
\hline Moraceae & Ficus carica & Figueira & $\mathrm{e}$ \\
\hline Moraceae & Morus nigra $\mathrm{L}$. & Amoreira & e \\
\hline Moraceae & Ficus pumila $\mathrm{L}$. & Unha de gato & $\mathrm{e}$ \\
\hline Moraceae & Artocarpus heterophyllus Lam & Jaca & e \\
\hline Moraceae & Ficus Benjamina & Ficus variegata & e \\
\hline Musaceae & Musa spp. & Banana & $\mathrm{e}$ \\
\hline Myrtaceae & Myrcia cauliflora Berg & Jaboticaba & $\mathrm{n}$ \\
\hline Myrtaceae & Psidium guajava $\mathrm{L}$. & Goiabeira & $\mathrm{n}$ \\
\hline Myrtaceae & Eugenia uniflora $\mathrm{L}$. & Pitanga & $\mathrm{n}$ \\
\hline Myrtaceae & Eugenia pyriformis Cambess & Uvaia & $\mathrm{n}$ \\
\hline Myrtaceae & Eugenia involucrata DC & Cerejinha & $\mathrm{n}$ \\
\hline Myrtaceae & Campomanesia xanthocarpa Berg & Gabiroba & $\mathrm{n}$ \\
\hline Myrtaceae & Psidium cattleyanum & Araça & $\mathrm{n}$ \\
\hline Orchidaceae & Phalaenopsis sp. & Orquidea & $\mathrm{e}$ \\
\hline Oxalidaceae & Averrhoa carambola $\mathrm{L}$. & Carambola & $\mathrm{e}$ \\
\hline Passifloraceae & Passiflora sp. & Maracujá & $\mathrm{n}$ \\
\hline Poaceae & Paspalum notatum Flügge. & Grama & $\mathrm{e}$ \\
\hline Poaceae & Saccharum officinarum L & Cana-de-açucar & e \\
\hline Poaceae & Eleusine indica (L.) Gaertn. & Capim da cidade & e \\
\hline Poaceae & Cymbopogon citratus & Capim-limão & e \\
\hline Portulacaceae & Portulaca grandiflora & Onze-horas & $\mathrm{n}$ \\
\hline Rosaceae & Eriobotrya japonica LINDL. & Nespera & $\mathrm{e}$ \\
\hline Rosaceae & Rosa Alba L. & Roseira & incerta \\
\hline Rosaceae & Prunus persica (L.) Batsch & Pessego & $\mathrm{e}$ \\
\hline Rubiaceae & Coffea arábica $\mathrm{L}$ & Café & e \\
\hline Rubiaceae & Gardenia jasminoides & Gardênia & e \\
\hline Rutaceae & Citrus sp. & Limoeiro & e \\
\hline Sapindaceae & Litchi chinensis Sonn. & Lichia & $\mathrm{e}$ \\
\hline Solanaceae & $\begin{array}{l}\text { Acnistus arborescens } \\
\text { (L.) Schlecht. }\end{array}$ & Marianeira & $\mathrm{n}$ \\
\hline Solanaceae & Lycopersicon esculentum Mill. & Tomate & $\mathrm{e}$ \\
\hline Verbenaceae & Duranta erecta L. & Pingo d'ouro & $\mathrm{n}$ \\
\hline
\end{tabular}




\begin{tabular}{lccc}
\hline Continuação... & & & \\
\hline Vitaceae & Vitis vinifera L. & Parreira & e \\
Zingiberiaceae & Zingiber officinale [Willd.] & Gengibre & e \\
\hline Total $=$ & & $\mathbf{1 1 5}$ \\
\hline
\end{tabular}

$\mathrm{n}=$ nativa; $\mathrm{e}=$ exótica e $\mathrm{i}=$ incerta.

Angeoletto et al. (2011) ao realizarem levantamento da vegetação em quintais de quatro bairros periféricos a região central da cidade de Sarandi, Paraná, e obteviram um índice de Shannon $\left(\mathrm{H}^{\prime}\right)=3.64$, e observaram que os proprietários tinham uma preferência por plantas relacionadas à alimentação humana, sendo em média 3,95 árvores frutíferas por quintal. Nos quintais pesquisados neste estudo, cerca de $48(42 \%)$ das plantas registradas também estavam relacionadas ao consumo humano, sendo frutas, condimentos ou medicinais. Comparando com outras áreas verdes urbanas, o presente resultado assemelha-se aos resultados de Souza et al (2004) que ao realizarem o censo de vegetação arbórea nas ruas do municipio de Jaú, São Paulo registrou 75 espécies, onde 48 (64\%) eram exóticas. Faria et al. (2007) estudando a arborização de vias públicas da área central do município de Jacareí, São Paulo, encontraram uma proporção de 1:1 de árvores nativas e exóticas, sendo parte delas frutíferas. $\mathrm{O}$ fato das árvores plantadas nos quintais serem exóticas parece não representar um problema para a visitação de espécies de aves nativas. MacGregor-Fors (2008) em seu estudo realizado dentro da Universidade de Gualajara, México, localizada em um bairro considerado o ponto medio entre a área urbana e a área de mata, analisou a relação entre atributos de habitat urbano e a riqueza de aves no. Para tanto o autor estabeleceu oito parcelas na área de transição entre área urbana e mata. Os resultados mostraram queduas das oito parcelas apresentaram maior riqueza de aves (28 aves) e abrigavam quase exclusivamente uma espécie australiana a Grevillea robusta.

Quanto a avifauna o censo realizados nos quintais residenciais resultou em 31 espécies, pertencentes a 15 famílias (Quadro 2).

Os índices descritivos obtidos da comunidade foram: índice de diversidade de Shannon = 2.51, equabilidade $=0.39$ e dominância $=0.12$. O levantamento realizado por meio do conhecimento dos proprietários resultou em uma lista contendo 58 espécies, pertencentes a 23 famílias, sendo que 19 foram identificadas apenas a morfo-espécie. Os índices obtidos a partir das informações dos proprietários foram: diversidade de Shannon $=3.56$, equabilidade $=0.87$ e dominância $=0.04$.

O levantamento realizado a partir das informações fornecidas pelo proprietário revelou quase o dobro do número de aves observadas in loco. Uma das explicações para esse resultado é o tempo de moradia. Os proprietários registraram espécies que foram observadas ao longo de aproximadamente 30 anos. Em meados dos anos de 1980 a cidade de Jacareí tinha uma população de 115.738 habitantes, sendo que desse total, 107.860 viviam na área urbana (IBGE, 2010). Neste período a cidade ainda não era fortemente urbanizada.

O período amostral também pode ter influenciado em um menor número de espécies registradas, entretanto algumas espécies citadas pelos proprietários apresentam baixa probabilidade de ocorrência hoje, como jacuguaçu e tucano-de-bico-verde. Dessa forma é esperado que a lista de aves do proprietário fosse mais rica que a do observador.

Belaire et al. (2015), verificaram que a idade do morador foi muitas vezes relacionada com a percepção positiva de aves. Bjerke e Ostdahl (2004) estudaram as atitudes de moradores urbanos para com os animais sinantrópicos e sua participação em atividades orientadas para observação dos animais na cidade de Trondheim, Noruega, e verificaram que o aumento do interesse por observar aves era proporcional ao aumento da idade dos moradores. Já para Davies et al. (2012) que utilizaram dois conjuntos de dados de pesquisa de nível doméstico recolhidos de toda a Grã-Bretanha, analisaram se, e como o contexto 
socioeconômico de uma participação doméstica influenciava no fornecimento de alimentos para as aves silvestres e ao final registraram uma relação positiva entre a idade dos moradores e o interesse por alimentar as aves. Assim a riqueza de espécies relatada pelo proprietário neste estudo pode estar diretamente relacionada tanto com a idade como o tempo de moradia.

Quadro 2. Lista das espécies e seus valores de abundância e abundância relativa mensuradas nos quintais residenciais na área urbana de Jacareí (SP).

\begin{tabular}{|c|c|c|c|c|c|}
\hline Família & Espécie ave/Nome vulgar & Origem & $\begin{array}{l}\text { Nível } \\
\text { trófico }\end{array}$ & Abundância & $\begin{array}{l}\text { Abundância } \\
\text { Relativa }\end{array}$ \\
\hline Columbidae & Columba livia (Gmelin, 1789) pombo-doméstico & $\mathrm{E}$ & $\mathrm{G} / \mathrm{F}$ & 31 & 0.0433 \\
\hline Columbidae & Columbina talpacoti (Temminck, 1811) rolinha & $\mathrm{N}$ & $\mathrm{G} / \mathrm{F}$ & 82 & 0.1145 \\
\hline Columbidae & Zenaida auriculata (Des Murs, 1847) avoante & $\mathrm{N}$ & $\mathrm{G}$ & 9 & 0.0126 \\
\hline Columbidae & $\begin{array}{l}\text { Patagioenas picazuro (Temminck, 1813) asa- } \\
\text { branca }\end{array}$ & $\mathrm{N}$ & $\mathrm{G} / \mathrm{F}$ & 2 & 0.0028 \\
\hline Trochilidae & $\begin{array}{l}\text { Chlorostilbon lucidus (Shaw, 1812) besourinho- } \\
\text { de-bico-vermelho }\end{array}$ & $\mathrm{N}$ & $\mathrm{N}$ & 2 & 0.0028 \\
\hline Trochilidae & $\begin{array}{l}\text { Amazilia fimbriata (Gmelin, 1788) beija-flor-de- } \\
\text { garganta-verde }\end{array}$ & $\mathrm{N}$ & $\mathrm{N}$ & 7 & 0.0098 \\
\hline Trochilidae & $\begin{array}{l}\text { Amazilia lactea (Lesson, 1832) beija-flor-de- } \\
\text { peito-azul }\end{array}$ & $\mathrm{N}$ & $\mathrm{N}$ & 4 & 0.0056 \\
\hline Trochilidae & $\begin{array}{l}\text { Eupetomena macroura (Gmelin, 1788) beija- } \\
\text { flor-tesoura }\end{array}$ & $\mathrm{N}$ & $\mathrm{N}$ & 28 & 0.0391 \\
\hline Picidae & $\begin{array}{l}\text { Picumnus cirratus (Temminck, 1825) } \\
\text { picapauzinho-barrado }\end{array}$ & $\mathrm{N}$ & I & 1 & 0.0014 \\
\hline Psittacidae & $\begin{array}{l}\text { Psittacara leucophthalmus (Statius Muller, } \\
\text { 1776) periquitão }\end{array}$ & $\mathrm{N}$ & $\mathrm{G} / \mathrm{F}$ & 4 & 0.0056 \\
\hline Furnariidae & Furnarius rufus (Gmelin, 1788) joão-de-barro & $\mathrm{N}$ & I & 3 & 0.0042 \\
\hline Rhynchocyclidae & $\begin{array}{l}\text { Todirostrum cinereum (Linnaeus, 1766) } \\
\text { ferreirinho-relógio }\end{array}$ & $\mathrm{N}$ & I & 1 & 0.0014 \\
\hline Tyrannidae & $\begin{array}{l}\text { Serpophaga subcristata (Vieillot, 1817) } \\
\text { alegrinho }\end{array}$ & $\mathrm{N}$ & I & 3 & 0.0042 \\
\hline Tyrannidae & Pitangus sulphuratus (Linnaeus, 1766) bem-te-vi & $\mathrm{N}$ & $\mathrm{O}$ & 33 & 0.0461 \\
\hline Tyrannidae & $\begin{array}{l}\text { Camptostoma obsoletum (Temminck, 1824) } \\
\text { risadinha }\end{array}$ & $\mathrm{N}$ & I & 4 & 0.0056 \\
\hline Tyrannidae & Tyrannus melancholicus (Vieillot, 1819) suiriri & $\mathrm{N}$ & I & 11 & 0.0154 \\
\hline Tyrannidae & Tyrannus savana (Vieillot, 1808) tesourinha & $\mathrm{N}$ & I & 1 & 0.0014 \\
\hline Tyrannidae & Megarynchus pitangua (Linnaeus, 1766) neinei & $\mathrm{N}$ & I & 1 & 0.0014 \\
\hline Troglodytidae & Troglodytes musculus (Naumann, 1823) corruíra & $\mathrm{N}$ & I & 45 & 0.0628 \\
\hline Turdidae & Turdus leucomelas (Vieillot, 1818) sabiá-branco & $\mathrm{N}$ & $\mathrm{I} / \mathrm{F}$ & 49 & 0.0684 \\
\hline Turdidae & Turdus rufiventris Vieillot, 1818 sabiá-laranjeira & $\mathrm{N}$ & $\mathrm{I} / \mathrm{F}$ & 2 & 0.0028 \\
\hline Mimidae & $\begin{array}{l}\text { Mimus saturninus (Lichtenstein, 1823) sabiá-do- } \\
\text { campo }\end{array}$ & $\mathrm{N}$ & $\mathrm{I} / \mathrm{F}$ & 8 & 0.0112 \\
\hline Icteridae & Chrysomus ruficapillus (Vieillot, 1819) garibaldi & $\mathrm{N}$ & $\mathrm{O}$ & 10 & 0.0140 \\
\hline Thraupidae & Coereba flaveola (Linnaeus, 1758) cambacica & $\mathrm{N}$ & $\mathrm{N} / \mathrm{F}$ & 32 & 0.0447 \\
\hline Thraupidae & Tangara preciosa (Cabanis, 1850) saíra-preciosa & $\mathrm{N}$ & $\mathrm{F} / \mathrm{I}$ & 1 & 0.0014 \\
\hline Thraupidae & Tangara cayana (Linnaeus, 1766) saíra-amarela & $\mathrm{N}$ & $\mathrm{F} / \mathrm{I}$ & 17 & 0.0237 \\
\hline Thraupidae & $\begin{array}{l}\text { Tangara sayaca (Linnaeus, 1766) sanhaço- } \\
\text { cinzento }\end{array}$ & $\mathrm{N}$ & $\mathrm{F} / \mathrm{I}$ & 110 & 0.1536 \\
\hline Thraupidae & $\begin{array}{l}\text { Tangara palmarum (Wied, 1823) sanhaço-do- } \\
\text { coqueiro }\end{array}$ & $\mathrm{N}$ & $\mathrm{F} / \mathrm{I}$ & 6 & 0.0084 \\
\hline Fringillidae & Euphonia chlorotica (Linnaeus, 1766) fim-fim & $\mathrm{N}$ & $F / I$ & 2 & 0.0028 \\
\hline Estrildidae & Estrilda astrild (Linnaeus, 1758) bico-de-lacre & $\mathrm{E}$ & $\mathrm{G}$ & 14 & 0.0196 \\
\hline Passeridae & Passer domesticus (Linnaeus, 1758) pardal & $\mathrm{E}$ & $\mathrm{O}$ & 193 & 0.2696 \\
\hline Total & & & & 716 & \\
\hline
\end{tabular}

G= Granívoro; F= Frugívoro; N= Nectarívoro; I= Insetívoro; O= Onívoro. E= Exótico e N= Nativo 
As espécies mais conhecidas e citadas pelos proprietários foram: pardal, rolinha e bemte-vi. Entre as mais raras e inusitadas estão o tucano-de-bico-verde e jacuguaçu. Ao serem perguntados se havia algum tipo de ave da qual não gostavam 55\% dos proprietários responderam que sim, sendo a ave mais citada o pombo-doméstico, em 59\% das respostas. Sete proprietários alegaram ter problemas com aves em suas residências, destes quatro citaram pombo-doméstico como sendo causa de problemas, e citaram a presença de carrapatos, pulgas e piolhos como provenientes da presença de aves, e apenas um proprietário disse ter problemas com fezes de aves no quintal.

Nunes (2003) relata que parasitas como piolhos de pombos, ácaros, percevejos e carrapatos infestam tanto os pombos como seus ninhos, abrigos e locais com grande concentração destas aves. De acordo com Magnino et al. (2009) o aumento das populações de pombos é causa de preocupação na Europa devido ao efeito prejudicial de fezes de pombos sobre a higiene ambiental.

A segunda ave mais citada como indesejável, o pardal, é uma espécie capaz de aproveitar os recursos oriundos da produtividade do ecossistema humano de forma direta, em função da capacidade dessa espécie de aproveitar esses recursos. Ssendo assim o pardal é comum em vários centros urbanos do Brasil e do mundo (Johnston e Janiga 1995, Argel-de-Oliveira, 1996; Leveau e Leveau, 2004; Lim e Sodhi, 2004, Vincent 2005). A rejeição ao pardal não esteve relacionada a zoonoses e sim devido a abundãncia desta espécie no quintal.

Quanto à impermeabilização foi observado que nos quintais não impermeabilizadoo número de espécies de aves foi significativamente diferente $(\mathrm{KW}=8.380 ; \mathrm{p}=0,038)$ das áreas que tinham mais de $50 \%$ da superfície impermeabilizada (Figura 2), seja por cimento ou piso. O número de espécies é inversamente proporcional à impermeabilização do solo. MacgregorFors e Schondube (2012) já haviam registrado esse efeito visto que a medida em que ocorreu o aumento da impermeabilização do solo, houve também a diminuição principalmente da abundância de aves como também diminuição da riqueza e diversidade.

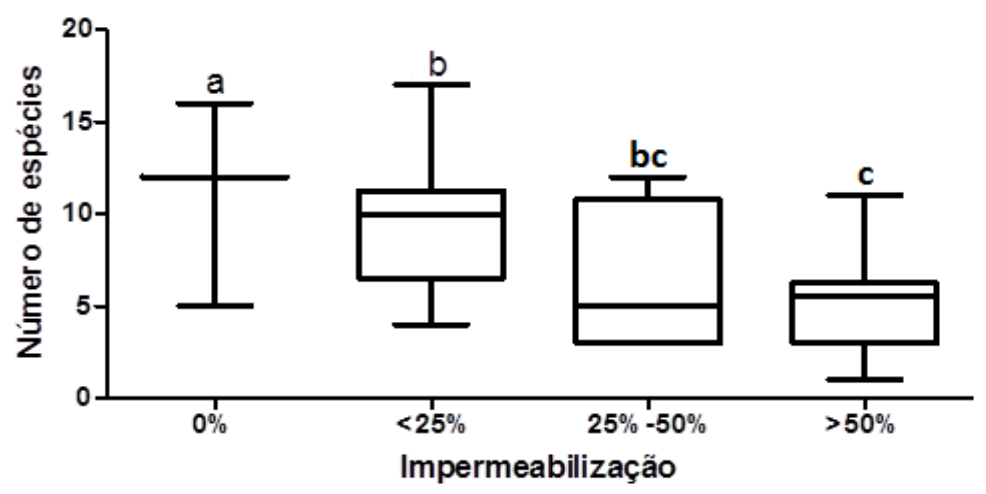

Figura 2. Média, desvio padrão e valores máximos e mínimos do número de espécies de aves em função da área impermeabilizada dos quintais em residências urbanas em Jacareí, SP. Letras diferentes indicam diferenças entre as médias $(\mathrm{p}<0,05)$.

Quanto as variáveis estudadas referentes as modificações produzidas pelo proprietário, foram obtidos diferentes resultados, mas todos confirmam que tais modificações influenciaram a distribuição da comunidade de aves. Estudos de Daniels e Kirkpatrick (2006) evidenciaram a interferência da variação de jardins residenciais sobre comunidades de aves urbanas em dez subúrbios de Hobart, Tasmânia, Austrália.

Quanto à presença de alimentadores, cinco (16\%) proprietários participantes do estudo afirmaram disponibilizar alimentos para as aves, preferncialmente frutas, sementes ou restos de comida. De modo geral a riqueza de aves foi significativamente diferente, $(U=21 ; p=0,02)$ 
entre as residências que ofereciam alimentos regularmente, sendo que a média de espécies de aves foi 9,8 $\pm 1,3$. Nas residências que não ofereciam alimentos a média foi $6,8 \pm 3,0$ de espécies de aves. Para Parsons et al. (2006) o fornecimento de carne e sementes foram associados com um aumento na abundância total de aves, mais especificamente, a abundância de espécies de aves pequenas foi menor nos jardins em que a carne era fornecida. Os mesmos autores referem que a suplementação alimentar pode tornar o quintal mais atraente como pode simplesmente refletir a alta diversidade já existente em torno do local. No estudo de Fuller et al. (2007) a presença de comedouros não teve influencia na riqueza de espécies, porém na abundância houve influência de $47 \%$, mas principalmente para as espécies menos exigentes, isto é, aquelas mais ajustadas ao ambiente e à presença humana.

Em relação aos animais de estimação, dos quintais amostrados $74 \%$ abrigavam animais de estimação como cães, gatos, gato+cão e cão+passarinho. A análise de comparação entre os quatro grupos mostrou que a abundância de aves foi menor onde havia a presença de gato $(\mathrm{KW}=9,09 ; \mathrm{p}=0,02)$. Três espécies de aves indicaram baixa probabilidade de persistência da população nos locais onde havia gato. Van Heezik et al. (2010) avaliando o impacto da presença de gatos domésticos sobre a persistência de populações de aves nativas e exóticas urbanas em Dunedin, Nova Zelândia, verificaram que mesmo os gatos tendo acesso a fragmentos de matas adjacentes, preferiam caçar nos jardins.

Stracey (2011), estudou a taxa de predação de ninhos em sete locais de estudo em Gainesville, Flórida, Estados Unidos da Ámerica, incluindo três bairros residenciais, encontrando que em habitats urbanos os gatos foram responsáveis por mais de 70\% (17 em 24) dos eventos de predação. O estudo de Belaire et al. (2014) mostrou que a presença de cães e gatos livres nos quintais pode reduzir a riqueza de aves, agindo como um elemento tanto de dissuasão na ocupação da área quanto de predação direta.

Neste estudo observou-se que os moradores mais antigos demonstraram uma maior preocupação e afinidade com o conhecimento do meio ambiente. Talvez exista nisso um indício da perda da diversidade cultural em relação ao conhecimento natural pelos mais jovens, tanto pelo tipo de habitação dos dias atuais, quanto pela rotina corrida do dia-a-dia. É uma questão que merece ser melhor estudada.

$\mathrm{O}$ fato de haver um grande número de plantas exóticas nos quintais parece não impedir que aves menos exigentes se utilizem dessas plantas para alimentação. Porém recomenda-se atenção e maior incentivo ao plantio de vegetação nativa nos centros urbanos, incluindo os quintais, para a atração da fauna nativa, sobretudo daquelas mais exigentes que acabam por perder habitat com a construção de grandes condomínios.

O controle das modificações realizadas pelo proprietário no quintal também poderia amenizar a diminuição de aves nativas nos centros urbanos, tais como: políticas públicas traçando parametros para quintais urbanos sustentáveis, com a redução da impermeabilização (o que já existe em muitas cidades); programas ambientais que incentivem as pessoas a observar e conhecer a avifauna urbana e a restrição de gatos livres nos jardins, tanto públicos como particulares.

\section{CONCLUSÃO}

Os proprietários mais idosos e com maior tempo de moradia no local têm um forte conhecimento da avifauna que visitam o quintal.

A composição de espécies de plantas, nativas e exóticas não influenciam na maior ou menor atrabilidade das aves, visto que as espécies de aves que frequentam os quintais urbanos são mais generalistas.

As modificações realizadas pelos proprietários em seus quintais podem tanto atrair como repelir as aves. Isto é, o aumento da área com vegetação, aumento do número de rvores, 
presença de alimentadores e ausência de inimigos naturais como os gatos podem melhorar a atrabilidade dos quintais para as aves.

\section{REFERÊNCIAS}

ALVARES, C. A. et al. Köppen's climate classification map for Brazil.Meteorologische Zeitschrift, v. 22, n. 6, p. 711-728, 2013. https://doi.org/10.1127/0941-2948/2013/0507

ANGEOleTtO, F. H. S.; SELEM, S.; DA SilVA MARTINS, N.; MORENO, M. Planejamento para o incremento da diversidade vegetal dos quintais de SarandiPr. SaBios Revista de Saúde e Biologia, v. 6, n. 2, 2011.

ARGEL-DE-OLIVEIRA, M. M. Aves urbanas. In: CONGRESSO BRASILEIRO DE ORNITOLOGIA, 5., 1996, Campinas. Anais... Campinas: UNICAMP, 1996. p. 151162.

BAGLIANO, R. V. Principais organismos utilizados como bioindicadores relatados com uso de avaliadores de danos ambientais. Revista Meio Ambiente e Sustentabilidade, v. 2, n. 1, p. 24-40, 2012.

BELAIRE, J. A.; WHELAN, C. J.; MINOR, S. Having our yards and sharing them too: the collective effects of yards on native bird species in an urban landscape. Ecological Applications, v. 24, n. 8, p. 2132-2143, 2014. http://dx.doi.org/10.1890/13-2259.1

BELAIRE, J. A. et al. Urban residents' perceptions of birds in the neighborhood: Biodiversity, cultural ecosystem services, and disservices. The Condor, v. 117, n. 2, p. 192-202, 2015. http://dx.doi.org/10.1650/CONDOR-14-128.1

BEUMER, C.; MARTENS, P. Biodiversity in my (back) yard: towards a framework for citizen engagement in exploring biodiversity and ecosystem services in residential gardens. Sustainability Science, p. 1-14, 2014. http://dx.doi.org/10.1007/s11625-014-0270-8

BJERKE, T.; OSTDAHL, T. Animal-related attitudes and activities in an urban population. Anthrozoos, v. 17, p. 109-129, 2004. http://dx.doi.org/10.2752/089279304786991783

BUTLER, D.; SPENCER, N. The century of the city. Nature, v. 467, 2010.

DANIELS, G. D.; KIRKPATRICK, J. B. Does variation in garden characteristics influence the conservation of birds in suburbia? Biological Conservation, v. 133, n. 3, p. 326335, 2006. http://dx.doi.org/10.1016/j.biocon.2006.06.011

DAVIES, Z. G.; FULlER, R. A.; DALliMER, M.; LORAM, A. e GASTON, K. J. Household factors influencing participation in bird feeding activity: A national scale $\begin{array}{llllllll}\text { analysis. PLOS One, v. 7, n. 6, p. } & \text { e 39692, }\end{array}$ http://dx.doi.org/10.1371/journal.pone.0039692

FARIA, J. L. G.; MONTEIRO, E. A.; FISCH, S. T. V. Arborização de vias públicas do município de Jacareí-SP. Revista da sociedade brasileira de arborização urbana, $v$. 2, n. 4, p. 20-33, 2007.

FERNÁNDEZ-JURICIC, E. Avifaunal use of wooded streets in an urban landscape. Conservation Biology, v. 14, n. 2, p. 513-521, 2000. http://dx.doi.org/10.1046/j.15231739.2000.98600.x

FERREIRA, A. O processo de metropolização do espaço no Estado do Rio de Janeiro e os projetos de revitalizações: mais do mesmo. GeoPuc, v. 4, p. 17-52, 2011. 
FULLER R. A.; WARREN P. H.; ARMSWORTH P. R.; BARBOSA, O.; GASTON K, J. Garden bird feeding predicts the structure of urban avian assemblages. Diversity and Distributions, v. 14, p. 131-137, 2007. http://dx.doi.org/10.1111/j.14724642.2007.00439.x

GALLUZZI, G.; EYZAGUIRRE, P.; NEGRI, V. Home gardens: neglected hotspots of agrobiodiversity and cultural diversity. Biodiversity and Conservation, v. 19, n. 13, p. 3635-3654, 2010. http://dx.doi.org/10.1007/s10531-010-9919-5

INSTITUTO BRASILEIRO DE GEOGRAFIA E ESTATÍSTICA. Cidades: Jacareí. 2010. Disponível em: https://goo.gl/Q7FDxS. Acesso em: 13 nov. 2014

INSTITUTO BRASILEIRO DE GEOGRAFIA E ESTATÍSTICA. Manual técnico da vegetação brasileira. 2. ed. Rio de Janeiro, 2012. 271p.

JOHNSTON, R. F.; JANIGA, M. Feral pigeons. New York: Oxford University, 1995.

LEVEAU, L. M.; LEVEAU, C. M. Comunidades de aves en un gradiente urbano de la ciudad de Mar del Plata, Argentina. El hornero, v. 19, n. 1, p. 13-21, 2004.

LIM, H. C.; SODHI, N. S. Responses of avian guilds to urbanisation in a tropical city. Landscape and Urban Planning, v. 66, n. 4, p. 199-215, 2004. http://dx.doi.org/10.1016/S0169-2046(03)00111-7

LORENZI, H. Plantas para jardim no Brasil: herbáceas, arbustivas e trepadeiras. Nova Odessa: Instituto Plantarum, 2013. 1120 p.

LUCHIARI, A. Identificação da cobertura vegetal em áreas urbanas por meio de produtos de sensoriamento remoto e de um sistema de informação geográfica. Revista do departamento de geografia, v. 14, p. 47-58, 2001. http://dx.doi.org/10.7154/RDG.2001.0014.0005

MACGREGOR-FORS, I. Relation between habitat attributes and bird richness in a western Mexico suburb. Landscape and urban planning, v. 84, n. 1, p. 92-98, 2008. http://dx.doi.org/10.1016/j.landurbplan.2007.06.010

MACGREGOR-FORS, I.; SCHONDUBE, J. E. Urbanizando La naturaleza: cambiosen las comunidades de aves asociadas com as entamientos humanos pequeños. Revista mexicana de biodiversidad, v. 83, n. 2, p. 477-486, 2012.

MAGNINO, S. et al. Chlamydial infections in feral pigeons in Europe: Review of data and focus on public health implications. Veterinary microbiology, v. 135, n. 1, p. 54- 67, 2009. http://dx.doi.org/10.1016/j.vetmic.2008.09.045

MAGURRAN, A. E. Measuring biological diversity. New York: John Wiley \& Sons, 2013.

MELLES, S.; GLENN, S.; MARTIN, K. Urban bird diversity and landscape complexity: species--environment associations along a multiscale habitat gradient. Conservation Ecology, v. 7, n. 1, 2003.

MILLER, J. R. Biodiversity conservation and the extinction of experience. Trends in $\begin{array}{lllllllll}\text { Ecology } \quad \text { \& } & \text { Evolution, } & \text { v. } & 2005 . & \text { n. }\end{array}$ http://dx.doi.org/10.1016/j.tree.2005.05.013

MÜLLER N.; WERNER, P.; KELCEY, J. G. (Eds.) Urban biodiversity and design. Vol 7. Oxford: Wiley-Blackwell, 2010. 
NUNES, V. F. P. Pombos urbanos: o desafio de controle. Biológico, v. 65, n. 1/2, p. 89-92, 2003.

OPPLIGER, E. A.; FONTOURA, F. M.; OLIVEIRA, A. K. M.; TOLEDO, M. C. B.; SILVA, M. H. S.; GUEDES, N. M. R. Avifauna de três áreas verdes de Campo Grande, MS, uma sugestão de roteiro de observação de aves como produto agregador de valor turístico. Revista Brasileira de Pesquisa em Turismo, v. 10, p. 274-296, 2016.

PARSONS, H.; MAJOR, R. E.; FRENCH, K. Species interactions and habitat associations of birds inhabiting urban areas of Sydney, Australia. Austral Ecology, v. 31, n. 2, p. 217 227, 2006. http://dx.doi.org/10.1111/j.1442-9993.2006.01584.x

PIACENTINI, V. Q. et al. Annotated checklist of the birds of Brazil by the Brazilian Ornithological Records Committee. . Brazilian Journal of Ornithology, v. 23, n. 2, p. 90-298, 2015.

SOUZA, A. M. de; NACHTERGAELE, M. F.; CARBONI, M. Inventário florestal da arborização do município de Jaú/SP. Jaú: Instituto Pró-Terra; SEMEIA, 2004. Relatório técnico.

STRACEY, C. M. Resolving the urban nest predator paradox: The role of alternative foods for nest predators. Biological Conservation, v. 5, n. 144, p. 1545-1552, 2011. http://dx.doi.org/10.1016/j.biocon.2011.01.022

TOLEDO, M. C. B.; BATISTA, G.; MAIA, J. S. Avaliação das áreas verdes urbanas e da diversidade de aves do Município de Taubaté, SP. In: SIMPÓSIO BRASILEIRO DE SENSORIAMENTO REMOTO - SBSR, XV., 2011, Curitiba. Anais...São José dos Campos: INPE, 2011. p 3174.

VAN HEEZIK, Y. et al. Do domestic cats impose an unsustainable harvest on urban bird populations? Biological Conservation, v. 143, n. 1, p. 121-130, 2010. http://dx.doi.org/10.1016/j.biocon.2009.09.013

VINCENT, K. E. Investigating the causes of the decline of the urban house sparrow Passer domesticus population in Britain. 2005. Tese (Doutorado) - Montfort University, Leicester, 2005. 